\title{
HYMENOLEPIS NANA ET HYMENOLEPIS FRATERNA
}

\author{
Par Ch. JOYEUX
}

L'étude biologique d'Hymenolepis nana (V. Sieb. 1852) et d'Hymenolepis fraterna Stiles, 1906 (syn. Tænia murina Duj. 1845), ont donné lieu récemment à un certain nombre de travaux dont il nous paraît intéressant d'entretenir les lecteurs de ces Annales. On s'est efforcé d'éclaircir, d'une part leur développement, d'autre part, leur valeur spécifique. Pour plus de clarté, nous allons sérier les questions et envisager successivement le cycle évolutif d' $H$. fraterna, le cycle évolutif d'H. nana, l'identité ou la dualité de ces espèces.

1. Cycle Évolutif d'Hymenolepis fraterna. - Le cycle évolutif d'Hymenolepis fraterna, cestode commun des rats et des souris, existant aussi chez des rongeurs voisins, a été établi par Grassi et ses collaborateurs. Dans un important mémoire, Grassi et Rovelli (1892) l'ont exposé en détail avec figures à l'appui. Voici le résumé de leurs recherches : le cycle est direct ; le rat absorbe les œufs du ténia qui éclosent dans son intestin, l'embryon s'en échappe, pénètre dans les villosités intestinales et y prend la forme cysticercoïde. Au bout de trois à quatre jours, cette forme larvaire s'évagine, le jeune ver tombe dans la cavité intestinale et s'y développe. Cependant quelques auteurs (Dampf, Nicoll et Minchin, Johnston) ont découvert chez diverses puces des cysticercoïdes, dont le scolex ressemblait à celui d' $H$. fraterna et l'hypothèse a été émise que ces insectes pourraient être les hôtes intermédiaires du cestode. Reprenant ces expériences, je suis arrivé à confirmer les travaux de Grassi el de ses collaborateurs (1920), mais je n'ai pu réussir à infester les puces communes du rat: Ceratophyllus fasciatus (Bosc), Xenopsylla cheopis (Roths.), j'ai également essayé sans succès avec Pulex irritans L. ; mes tentatives ont été faites avec des larves et des adultes. J'ai done conclu que le cycle est direct comme l'avaient déjà vu les auteurs italiens.

Clayton Lane et George C. Low, dans un ouvrage classique (1923) critiquèrent assez vivement mes conclusions et n'admirent pas ce cycle direct. Je crus devoir protester contre ces critiques (1923) ; mes contradicteurs anglais (1923) reconnurent loyalement n'avoir pas lu mon travail définitif (1920), mais seulement une courte note préliminaire (1919) ; de là leur reproche injustifié à mon égard,

Annales de Parasitologie, T. III, N ${ }^{\circ} 3$. - Juillet 1925, p. 270-280. 
m'accusant d'avoir publié mes expériences sans donner de détails ; néanmoins ils maintinrent leurs conclusions.

Peu après, W. Nicoll (1923), publia une lettre de rectification à propos du cysticercoïde trouvé par lui et Minchin chez la puce, disant qu'ils n'avaient pas donné la diagnose d'H. nana comme certaine.

A la suite de cette discussion, le cycle évolutif d'H. fraterna fut étudié par divers auteurs.

H. Scott, reprenant les expériences de Grassi, les confirme pleinement (1923) et infeste des souris par voie directe en se mettant à l'abri des causes d'erreur expérimentales. II observe le développement des cysticercoïdes et dies vers adultes chez les animaux ayant ingéré des œufs de ténia. Dans une autre expérience, sans intérêt aujourd'hui, il montre qu'Hymenolepis longior Baylis, 1922, évolue aussi directement ; mais Baylis lui-même a reconnu ultérieurement (1924) que son $H$. longior n'est autre qu'H. fraterna. H. Scott répond en outre à une objection de Clayton Lane et George-C. Low, d'après laquelle les cysticercoïdes, formés dans les villosités seraient évacués avec les matières fécales et ingérés à nouveau par la souris. Il fait avaler des villosités intestinales contenant des cysticercoïdes par des souris neuves et n'observe aucun développement. D'autre part, il suit avec précision tous les stades de l'évolution intestinale et confirme les résultats de Grassi et les miens (1923-1924).

W.-N.-F. Woodland (1924 a) arrive aux mêmes conclusions : 20 souris neuves, soigneusement à l'abri de toute contamination, avalent des œufs d'H. fraterna. 19 s'infestent dans les délais habituels, une seule reste indemne. Woodland dit à ce propos que les très jeunes souris (very joung mice) ne sont pas sensibles à l'infestation; contrairement à ce que j'ai affirmé. Ceci n'est pas tout à fait exact. Les très jeunes souris s'infestent mal, en effet ; l'époque de choix, ai-je dit (1920, p. 44-45-163) est dans les 6 à 8 semaines qui suivent le sevrage. C'est d'ailleurs une règle générale en parasitologie que les jeunes animaux s'infestent plus facilement que les adultes.

Graybill (1924) confirme également les résultats précédents en infestant directement des souris.

Enfin, depuis la publication de mon travail, j'ai eu l'occasion de répéter plusieurs fois, dans un but d'enseignement, l'expérience en question. Elle réussit toujours, si les jeunes rats ne sont déjà pas porteurs d'H. fraterna, auquel cas ils ont acquis l'immunité, comme je l'avais déjà vu en 1920 .

Je pense que le cycle d'H. fraterna est donc bien démontré. J'ai d'ailleurs, depuis cette époque, signalé d'autres cestodes : Hymeno- 
lepis erinacei (Gmelin) du hérisson (1921), Cylindrotænia americana (Jewel) des grenouilles de Mozambique (1924), qui ont aussi une évolution directe. Remarquons que ce développement s'accomplit en réalité, au moins pour $H$. fraterna, en deux phases. Comme l'a très bien observé Scott, le cysticercoïde se trouve dans la partie médiane de l'intestin grêle, tandis que l'adulte se voit dans la dernière portion. L'intestin des rats blanes de laboratoire mesure généralement 95 centimètres de long, il est facile de lui donner ces dimensions en l'étirant ou en le rétractant légèrement à l'état frais. Les cysticercoïdes se trouvent habituellement du $50^{\circ}$ au $75^{\circ}$, les adultes du $73^{\circ}$ au $95^{\circ}$ centimètre. On peut donc considérer que le troisième quart de l'intestin grêle constitue un véritable hôte intermédiaire, tandis que le quatrième quart est l'hôte définitif. La migration du cestode persiste, mais condensée chez le même animal.

II. Cycle Évolutif d'Hymenolepis nana. - Le cycle évolutif d'Hymenolepis nana a été établi d'une façon assez grossière dès 1906 par Calandruccio. Faisant avaler des œufs d' $H$. nana à un enfant, il trouve, à partir des $19^{\circ}-25^{\circ}$ jours après l'infestation, des œufs dans les selles de ce sujet.

Goldman (1921) croit avoir vu des cysticercoïdes d' $H$. nana dans les selles d'un enfant.

Saeki (1920) obtient des résultats positifs en infestant des souris, des rats avec des œufs d'H. nana; il infeste également une fillette de 4 ans et tente l'expérience sur lui-même sans succès. Le développement est direct, l'évolution est la même que celle d'H. fraterna : onchosphères s'introduisant dans les villosités de la partie supérieure et moyenne de l'intestin grêle ; au $5^{\circ}$ jour, jeunes vers dans la partie postérieure ; au $16^{\circ}-17^{\circ}$ jour, apparition des œufs dans les selles. Au bout d'un mois ou plus, les parasites sont expulsés spontanément.

Uchimura (1922) infeste également le rat avec des œufs d'H. nana.

Les travaux de ces deux auteurs, publiés dans des périodiques japonais, ne sont connus en Europe que par les analyses du Tropical diseases Bulletin.

W.-N.-F. Woodland récemment $(1924 b)$ infeste des souris avec des œufs d' $H$. nana et confirme les travaux précédents; nous allons revenir sur cette expérience.

Donc le cycle évolutif d'H. nana est bien direct, comme celui d'H. fraterna du rat ; la transmission se fait sans hôte intermédiaire.

III. Unicité ou DUALITÉ D'Hymenolepis nana et d'Hymenolepis fraterna. - Depuis longtemps, cette question est discutée : V. Linstow, Moniez, R. Blanchard, Castellani sont dualistes ; 
Stiles a créé la variété fraterna pour désigner le cestode du rat (1906). Grassi et Rovelli, Lutz, Mingazzini, Massari sont unicistes.

Nous allons envisager successivement: la morphologie de ces ténias, les résultats des infestations expérimentales, la distribution géographique, la spécificité des Hymenolepis.

$1^{\circ}$. - Morphologie. - Elle est difficile à étudier pour $H$. nana de l'homme. En effet, ce cestode est souvent récolté dans des conditions défectueuses : évacué avec les matières fécales par un antihelmintique, ou trouvé à une autopsie faite plus ou moins longtemps après la mort. Dans les deux cas, il est altéré et souvent macéré. $H$. fraterna au contraire peut être recueilli dans d'excellentes conditions immédiatement après avoir sacrifié le rongeur qui l'héberge. L'examen est donc difficilement comparable. Je suis arrivé (1920), à trouver entre les deux vers de très légères différences dans la taille des anneaux et des ventouses, un peu plus grande chez $H$. fraterna; les tubercules de la coque interne des œufs, un peu mieux marqués chez $H$. fraterna. Mais, comme je viens de le dire, ces matériaux sont peu comparables ; en outre, ces légères différences provenant de moyennes, s'appliquent à des types morphologiques n'existant pas dans la nature, elles ne sauraient être utilisées pour la diagnose d'un individu isolé.

Ceci ne veut aucunement dire qu'il n'y a pas de différence entre les deux espèces ; mais simplement que dans l'état actuel de nos connaissances, on n'a pu en constater jusqu'à présent. Il est fort possible que l'examen d'organes sur lesquels l'attention des systématiciens n'est pas attirée actuellement, ou que l'étude de la structure histologique révèle des caractères distinctifs. Les anciens helminthologistes établissaient leurs diagnoses d'après les caractères extérieurs et ceux du, scolex ; actuellement nous étudions surtout l'anatomie des organes génitaux, des systèmes musculaire et aquifère ; il est probable que nos successeurs, perfectionnant nos méthodes, trouveront autre chose.

$2^{\circ}$. - Infestations expérimentales. - Looss relate incidemment une expérience négative en essayant d'infester Mus alexandrinus et Acomys cahirinus avec $H$. nana de l'homme. Stewart (1916) fait absorber sans succès ces œufs à deux rats. De même Malvoz, puis Schnell éprouvent des échecs dans des expériences analogues.

J'ai repris ces recherches (1920), en essayant d'infester 22 rongeurs avec des œufs d' $H$. nana de la façon suivante. 4 rats et 3 souris absorbent des œufs provenant des selles d'un indigène d'Afrique occidentale, 13 rats et 1 souris en absorbent d'autres provenant des selles d'une fillette arabe d'Alger, enfin 1 dernière souris ingère des œufs provenant des selles d'un enfant, dans le bassin minier 
de Liège. Aucun de ces animaux ne s'infeste. De plus, 6 rats de la deuxième expérience, la souris de la troisième ingèrent au bout de quatre et cinq jours de faibles doses d'œufs d'H. fraterna. La souris et 2 rats sur 6 montrent des formes de développement correspondant à la date de la deuxième infestation. Ce faible pourcentage est dû aux doses minimes absorbées. Les mêmes animaux, réfractaires à $H$. nana, sont donc sensibles à $H$. fraterna.

Woodland $(1924 b)$, relatant mon expérience, y fait plusieurs critiques auxquelles je vais répondre.

L'àge et la taille des animaux ne sont pas spécifiés. J'ai dit (p. 4445-163) que je choisissais les animaux peu après le sevrage. Quant à la taille qui ne présente que de faibles variations chez des rats et souris du même âge, je ne vois quelle importance elle peut avoir pour cette expérience.

La manière dont les animaux ont été infestés n'est pas spécifiée. J'ai indiqué ma technique (p. 48-155-156) et expliqué que les œufs étaient récoltés par centrifugation ou décantation. Je les ai fait absorber par voie buccale, et ne vois aucun détail complémentaire à fournir. C'est la technique habituellement employée en helminthologie pour infester un animal.

La nourriture des animaux en expérience n'est pas spécifiée. Elle a été indiquée (p. 40) : eau filtrée, aliments stérilisés, helminthologiquement parlant, soit : graines passées à l'autoclave, mie de pain (le pain ayant été stérilisé pendant sa cuisson, il suffit de prendre la partie intérieure).

Les 22 animaux n'étaient peut-être pas indemnes de cestodes. Ils étaient indemnes et j'ai expliqué la manière d'obtenir des animaux neufs (p. 39-40).

Il y a eu confusion dans les animaux infestés deux fois. J'ai eu soin de pratiquer ces deux infestations à des intervalles suffisamment éloignés pour éviter cette cause d'erreur que j'avais prévue : il est invraisemblable qu'on retrouve encore de jeunes vers n'ayant que les premiers anneaux formés au $11^{\circ}$ jour, et surtout des cysticercoïdes au huitième jour, l'évolution est beaucoup plus rapide.

En 1921, Goldman essaye sans succès d'infester des rats avec des œufs d'H. nana, de même Chandler en 1922. En 1923, Scott aboutit également à un échec, il est vrai que les œufs étaient peut-être altérés.

Telles sont les expériences négatives.

Voyons maintenant les résultats contraires.

Grassi fait avaler des œufs d' $H$. fraterna à des enfants qui, ultérieurement, montrent des œufs dans leurs selles. Mais ces sujets ont pu contracter $H$. nana, très fréquent dans le pays et l'auteur reconnaît lui-mème que son expérience n'est pas décisive. 
Nous avons vu plus haut que deux auteurs japonais, Saeki (1920) et Uchimura (1922) ont infesté des rats et souris avec des oufs d'H. nana. Malheureusement leurs travaux, publiés en japonais, sont inaccessibles aux lecteurs européens qui n'en ont connaissance que par les analyses du Tropical diseases Bulletin.

D'après Woodland $(1924$ b), Fülleborn aurait également réussi à infester des rats avec des œufs d'H. nana (communication orale).

Enfin Woodland $(1924 b)$ vient de reprendre ces recherches dans les circonstances suivantes. Prenant toutes précautions nécessaires pour éviter la contamination des animaux, cet auteur s'est rendu à Lisbonne, où l'H. nana est fréquent; les professeurs Machado et Bettencourt estiment que c'est le cestode humain le plus commun (1915-1916). Faisant avaler des œufs d'H. nana à 30 souris, il à obtenu une infestation chez 7 d'entre elles, soit 23,3 p. 100.

Ce pourcentage de succès est faible, comparé à celli qu'on obtient lorsqu'on infeste les rongeurs par $H$. fraterna, Woodland lui-même a trouvé 19 succès sur 20 souris $(1924 a)$. C'est également la proportion moyenne à laquelle j'arrive. Il explique son faible pourcentage par trois raisons :

A. - Les animaux étaient trop àgés. Il est difficile de critiquer cet argument. Cependant l'auteur, sachant que les animaux âgés s'infestent difficilement, a dû prendre ses précautions en conséquence.

B. - Les animaux, bien nourris, ne s'infestent pas facilement. Je suis d'un avis absolument opposé. Les jeunes animaux, dont le tube digestif est en bon état et la nutrition normale, offrent aux parasites intestinaux un meilleur milieu que lorsque l'alimentation est défectueuse et les fonctions digestives altérées. J'ai d'ailleurs relaté dans mon travail une petite expérience à ce sujet (1920, p. 164), que j'ai eu souvent l'occasion de confirmer depuis, avec divers helminthes.

C. - Ce faible pourcentage est dì à un changement d'hóte. Je suis pleinement d'accord avec Woodland pour cette dernière interprétation. Je pense, comme lui, qu' $H$. nana dérive sans doute d'une souche murine, mais qu'il s'est adapté à l'homme; c'est la raison pour laquelle $\mathrm{j}$ 'en ai fait une espèce biologique. Ce que Woodland a obtenu est une infestation expérimentale chez un hôte occasionnel, comme on en obtient continuellement dans les laboratoires. De ce qu'on a transmis un parasite ou un virus à un animal, il faut se garder de conclure que cet animal héberge normalement dans la nature le parasite ou le virus en question. Il serait excessivement facile de multiplier les exemples d'animaux de laboratoire auxquels on fait prendre des parasites dont ils n'ont jamais été porteurs dans 
la nature. Pour ne pas sortir du genre Hymenolepis, je rappelle que j'ai transmis au canard Hymenolepis serpentulus Schrank des passériformes (1920, p. 191); mais je n'ai aucunement conclu que ce cestode habitait normalement l'intestin des ansériformes.

Nous avons dit que dans l'expérience de Saeki, les $H$. nana développés expérimentalement chez le rat avaient été expulsés au bout d'un mois et plus. Cette longévité est-elle plus courte que celle d' $H$. fraterna? C'est encore un caractère qui s'observe fréquemment chez les parasites habitant des hôtes occasionnels. Ce point serait intéressant à éclaircir.

Les deux expériences de Woodland (1924 $a$ et $b$ ) sont très intéressantes en ce qu'elles nous montrent la différence de pourcentage entre l'infestation de l'hôte naturel et celle d'un hôte occasionnel. Il est regrettable que cet auteur en tire une conclusion uniciste; je pense au contraire qu'elles démontrent. la dualité des deux cestodes. Woodland fait remarquer que Grassi a eu de la difficulté à infester des rats sauvages en partant de l'Hymezolepis fraterna des rats de laboratoire. Il s'est sans doute adressé à des animaux trop âgés, ou mis en état de moindre résistance par la captivité, que les rats sauvages supportent souvent difficilement. J'ai fait de très nombreuses fois l'expérience inverse. Depuis le début de mes études sur ce sujet (1913), afin d'éviter la contamination possible des animaux neufs et aussi dans un but d'économie, je me procure le matériel nécessaire aux infestations chez les rats sauvages de Paris et de sa banlieue. C'est généralement $E$. norvegicus, plus rarement $E$. rattus, qui m'est apporté. Avec les $H$. fraterna provenant de l'un et de l'autre, j'ai infesté les diverses variétés de rats et de souris de laboratoire : blanc, gris, panaché, etc. J'ai toujours obtenu le même pourcentage élevé, à condition que les animaux soient en bonne santé et à l'àge favorable. La question de race ou de variété ne joue aucun rôle.

En résumé, $H$. fraterna se transmet au rat avec une grande facilité ; $H$. nana au contraire l'infeste difficilement. En totalisant les expériences des divers auteurs, on voit que les échees sont plus nombreux que les succès. Il faut donc admettre qu'il y a une différence entre les deux ténias et qu'un caractère biologique les distingue.

$3^{\circ}$. - Différences dans la répartition géographique. - Le manque de concordance dans la distribution géographique d'Hymenolepis fraterna et d'Hymenolepis nana est d'une extrême importance. Je renvoie à mon travail (1920) pour l'étude détaillée de cette répartition. On peut la résumer en disant qu'H. fraterna a été trouvé partout où on l'a cherché, il paraît cosmopolite; H. nana, au contraire, se voit dans les pays chauds, en Amérique du Sud et du Nord, en Europe méridionale et dans la région méditerranéenne où sa fré- 
quence est grande. Dans les pays froids, excepté les bassins miniers, il est inconnu ou extrêmement rare. Dans la région parisienne, par exemple, les rats (E. norvegicus) sont infestés dans la proportion de 157 sur 755 , soit 20,79 p. 100 . Cette statistique est faite sur des animaux de tous les âges. Le pourcentage serait plus élevé si elle portait exclusivement sur de jeunes rats. Jamais à ma connaissance, on n'a signalé $H$. nana. Woodland qui n'ignore pas ces faits prétend qu'ils n'ont aucune importance. Les examens de selles, dit-il, ne sont pas faits aussi fréquemment dans les pays froids que dans les pays chauds, parce que les maladies parasitaires intestinales y sont plus rares. C'est là une très grosse erreur; la pathologie intestinale moderne attache une grande importance aux analyses microscopiques de matières fécales, non seulement dans un but parasitologique, mais encore pour étudier les résidus de la digestion. Si $H$. nana existait à Paris, il aurait été depuis longtemps mis en évidence par les nombreux examens de selles pratiqués chaque jour dans les hôpitaux d'enfants et dans les laboratoires d'analyses. En Angleterre, il en est probablement de même; $H$. fraterna paraît s'y trouver communément, à en juger par la facilité avec laquelle les auteurs anglais ont pu se le procurer pour réaliser leurs infestations; H. nana doit y être inconnu ou au moins rare, sans quoi Woodland aurait pu faire ses expériences sur place sans avoir besoin de se transporter à Lisbonne.

Cet auteur donne aussi comme argument que les rats et souris sont moins communs dans les pays froids et vivent moins en promiscuité avec l'homme que dans l'Europe méridionale et les pays chauds. C'est là encore une affirmation sans preuves. Toutes les personnes habitant Paris savent combien les rongeurs y sont abondants, au point qu'ils ont constitué, il $\mathrm{y}$ a trois et quatre ans, un véritable danger social contre lequel on a dû prendre des mesures.

En résumé, si l'espèce du rat se transmettait à l'homme, la distribution géographique devrait être la même chez ses deux hôtes, ce qui n'est pas le cas.

$4^{\circ}$. - Spécificité des Hymenolepis. - La spécificité des cestodes, tout au moins des Cyclophyllides, vis-à-vis de leurs hôtes, est un - fait bien connu. Dans leur réplique à ma protestation, Clayton Lane et George Low (1923) qualifient mes idées de révolutionnaires (revolutionary views); parce que je fais entrer la notion d'hôte dans la détermination du parasite. Si l'on veut bien se reporter aux vieux ouvrages de Rudolphi et de Dujardin, on verra l'importance qu'ils attachaient à l'hòte pour la détermination des helminthes. Krabbe, à propos des ténias d'oiseaux (1869), remarque aussi que les divers groupes ornithologiques ont des 
cestodes différents ; O. Fuhrmann (1908) a très justement insisté sur la spécificité des ténias d'oiseaux, contrastant avec l'ubiquité dés cestodes inférieurs de poissons (1903). Cet auteur a mis en lumière que "chaque groupe d'oiseaux a ses formes de ténias qui lui appartiennent en propre ". Tout récemment, J.-G. Baer, se basant sur ces données, a pu établir que des cestodes indiqués par erreur comme provenant du cheval, étaient en réalité des parasites de la pintade (1924). Mes idées sur la spécificité n'ont donc rien de révolutionnaire, ce sont celles qui ont toujours été admises par des helminthologistes dont le nom fait autorité.

Pour les mammifères, il en est exactement de même que pour les ciseaux ; non seulement les grands ordres : ruminants, earnivores, etc., n'hébergent pas les mêmes cestodes, mais encore, dans les subdivisions d'un mème ordre, on trouve des ténias distincts, parfois limités à quelques genres voisins. Chez les rongeurs, par exemple, les ténias des Léporidés sont différents de ceux des Muridés. Je crois inutile d'insister sur ces faits élémentaires. Or le genre Hymenolepis représente un Cyclophyllide très évolué : réduction du nombre des crochets, condensation des organes génitaux, cycle évolutif réduit à un seul hôte pour quelques espèces, dont celles qui nous occupent. Il est invraisemblable, en l'état actuel de nos connaissances, de supposer qu'un Hymenolepis peut vivre indifféremment chez deux êtres aussi éloignés qu'un homme et un rat. Une telle ubiquité supposerait que cet Hymenolepis peut être hébergé par une foule de mammifères, or il n'en est pas ainsi. Dans la nature, on l'observe chez les animaux suivants : Rat : $E$. norvegicus et $E$. rattus; souris : M. musculus ; rats et souris de laboratoire ; mulot : M. sylvaticus ; $M$. pumilus ; lérot : E. quercinus. Je suis arrivé à le faire développer expérimentalement chez le campagnol : Microtus arvalis. Par contre, je n'ai pas pu réussir l'infestation avec le loir : Myoxus glis, ni avec le spermophile : Citillus citillus. Ransom a observé un cas de parasitisme spontané chez un cobaye ayant vécu en promiscuité avec des rats. C'est le premier cas signalé chez le cobaye, cependant fréquemment autopsié dans les laboratoires (1921). Les singes ne paraissent pas sensibles; H. Scott a échoué (1923) avec Macacus rhesus, j’ai également essayé l'infestation sans succès de Cercopithecus callitrichus, Chœropithecus sphinx, Macacus cynomolgus, Hamadryas hamadryas.

En définitive, $H$. fraterna vit dans la nature chez quelques rongeurs : rats, souris, mulots, lérots ; expérimentalement on peut le transmettre à des rongeurs voisins.

Si $H$. fraterna et $H$. nana avaient été trouvés chez deux oiseaux ou deux mammifères quelconques, appartenanf à des groupes diffé- 
rents, la question serait tranchée depuis longtemps et on en aurait fait des espèces distinctes. Mais, en raison de son intérêt médical, cette étude a été abordée par de nombreux auteurs, dont quelquesuns n'étaient peut-être pas suffisamment familiarisés avec la systématique et surtout la biologie des cestodes.

Lorsque nos connaissances, rudimentaires à l'heure actuelle, sur le chimisme intestinal et sur la nutrition des cestodes, auront fait des progrès, nous comprendrons peut-être pourquoi tel ténia a besoin, pour se développer, de se trouver dans l'intestin de tel hôte, pourquoi il doit se fixer dans telle portion de cet intestin, etc. Nos déterminations actuelles, basées sur l'anatomie d'animaux conservés dans l'alcool, paraittront probablement simplistes à nos successeurs. Tenons au moins compte dans nos diagnoses des quelques notions de biologie que nous possédons sur ces parasites, et dont une des plus précieuses est justement leur habitat.

En résumé, $H$. nana de l'homme, $H$. fraterna des muridés forment deux espèces biologiques comme le prouvent la façon différente dont ils se comportent dans les infestations expérimentales, et leur distribution géographique non concordante; ces faits sont en rapport avec nos connaissances zoologiques sur la spécificité du groupe de cestodes auquel appartient le genre Hymenolepis. Dans l'état actuel de nos connaissances, on n'a pu relever que d'insignifiantes différences morphologiques entre les deux espèces, mais l'une des deux (H. nana) est généralement récoltée dans de mauvaises conditions ; rien ne prouve qu'on ne découvrira pas plus tard des caractères distinctifs entre ces Hymenolepis.

$H$. nana et $H$. fraterna évoluent tous deux sans hôte intermédiaire, comme plusieurs autres cestodes.

\section{BibLiographie (1)}

Bafr (J.-G.). - Quelques cestodes d'oiseaux nouveaux et peu connus. Bull. Soc. Neuchâteloise des Sciences nal., XLIX, 1924, p. 138-153.

Baysis (H.-A.). - The range of variation of Hymenolepis nana in rats and mice. Parasilology, XVI, 1924, p. 415-418.

Cuandern (A.-C.). - Species of Hymenolepis as human parasites. Journ. Amer. med. assoc., LXXVIII, 1922, p. $(36$.

Chayton-Lane et Low (George-C.), in Byam et Arcaibald. - The praclise of Medicine in the Tropics, III, p. 1821-1824. Oxford med. Public., 1923.

Clayton-Lane et Low (George-C.). - Hymenolepis nana and Hymenolepis fraterna. A reply to M. Joyeux. Trans. Roy. Soc. Trop. Med. and Hyg., XVI, 1923, p. $504-508$.

(1) Les indications bibliographiques non mentionnées ci-dessus se trouvent dans mon travail de 1920 . 
Funruans (O.). - L'évolution des ténias et en particulier de la larve des Ichtyoténias. Soc. neuchateloise des Sciences naturelles, 22 mai 1903.

Goldman (A.). - Hymenolepis nana: possible cercocystis stage. Résumé in Journ. of trop. med. and hyg., XVIII, 1921, p. 217-218.

Joyeux (Ch.). - Hymenolepis nana (v. Siebold, 1852) et Hymenolepis nana var, fralerna Stiles, 1906. Bull. Soc. path. exot., XII, 1919, p. 228-231.

- Cycle évolutif de quelques cestodes. Recherches expérimentales. Bull. biol. France el Belgique, suppl. II, 1920.

- Développement direct d'un Hymenolepis (Téniadés) dans les villosités intestinales du hérisson. Bull. Soc. path. exot., XIV, 1921, p. 386-390.

- Hymenolepis nana v. Sieb. and Hymenolepis fraterna Stiles. Trans. Roy. Soc. Trop. Med. and Hyg., XVI, 1923, p. 502-504.

- Recherches sur le cycle évolutif des Cylindrotinia. Annales de Parasitologie, II, 1924, p. 7t-81.

Nicol. (W.). - The life-history of the tapeworm Hymenolepis nana. Lancet. 21 avril 1923, p. 82.

Ransom (B.-H.). - Helminthological Soc. of Washington, 23 octobre 1920. Journ. of Parasilolog! !, VII, 1921, p. 188.

SAEKI (Y.). - Analyse in Trop. dis. Bull., XVIII, 1921, p. 112.

ScotT (H.). - A contribution to the experimental study of the life-historie, of Hymenolepis fraterna Stiles, 1906, and Hymenolepis longior Baylis, 1922, in the mouse. Journal of Helminthology, I, 1923, p. 193-196.

- Stages in the direct development of Hymenolepis longior Baylis. Journal of Helminthology, 1I, 1924, p. 173-174.

Stewart (F.-H.). - Studies in Indian Helminthology. No III. Rec. Ind. Mus., XII, 1916, p. 295.

Uchimura (R.). - Analysé in Trop. dis. Bull., XX, 1923, p. 626.

Woodland (W.-N.-F.). - (a) On the Life-cycle of Hymenolepis fralerna (H. nana

var. fraterna Stiles) of the white mouse. Parasitology, XVI, 1924, p. 69-83.
(b) On the development of the human Hymenolepis nana (Siebold, 1852) in the white mouse; with remarks on $H$. fraterna, $H$. longior and $H$. diminuta. Parasitology, XVI, 1924, p. 424-435.

Laboratoire de Parasilologie de la Faculté de médecine de Paris. 